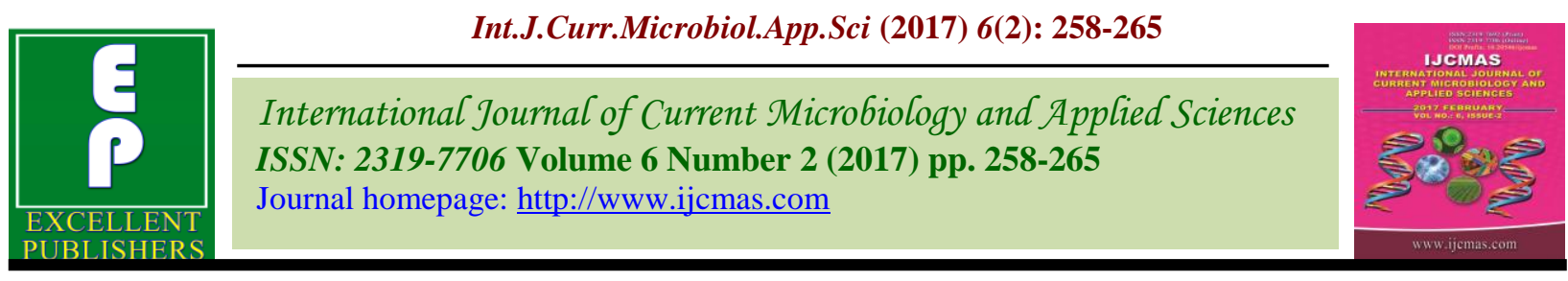

Original Research Article http://dx.doi.org/10.20546/ijcmas. 2017.602.031

\title{
Antagonistic Action of Trichoderma Isolates against Fusarium oxysporum f. sp. lycopersci
}

\author{
Akash Tomar ${ }^{1 *}$, Lakshman Prasad ${ }^{2}$, Abhishek Mishra ${ }^{1}$ and Sushma Sagar ${ }^{2}$ \\ ${ }^{1}$ Chandra Shekhar Azad University of Agriculture and Technology, Kanpur - 208002, Uttar \\ Pradesh, India \\ ${ }^{2}$ Division of Agriculture Chemicals, IARI, New Delhi-12, India \\ *Corresponding author
}

\begin{tabular}{|c|c|}
\hline & A B S T R A C T \\
\hline Keywords & \multirow{4}{*}{$\begin{array}{l}\text { In the present study, } 25 \text { soil samples were collected from the different location } \\
\text { of U.P and M.H. and } 17 \text { Trichoderma isolates were obtained belonging to three } \\
\text { different species T. harzianum, T. viride and T. koningi. All the obtained } \\
\text { isolates were studied microscopically for their species level identification. } \\
\text { Morphological characterization of all the isolates was done. All the isolates } \\
\text { were screened against Fusarium oxysporum } \mathrm{f} \text {. sp. lycopersci for their efficacy } \\
\text { through dual culture technique.nHighest mycelium sinhibition } \text { was } 0 \text { recorded } \\
\text { with SVPU-Thar7, }(81.40 \%) \text { and minimum with SVPU-Thar4 }(57.05 \%) \text {. }\end{array}$} \\
\hline $\begin{array}{l}\text { Trichoderma, } \\
\text { Biocontrol, } \\
\text { Fusarium, } \\
\text { Tomato }\end{array}$ & \\
\hline Article Info & \\
\hline $\begin{array}{l}\text { Accepted: } \\
\text { 12 January } 2017 \\
\text { Available Online: } \\
\text { 10 February } 2017\end{array}$ & \\
\hline
\end{tabular}

\section{Introduction}

Modern agricultural practices are getting affected by various problems such as disease, pest, drought, decreased soil fertility etc. due to the increasing use of chemical pesticides. The fungal disease are one of the major cause of crop productivity lose in India. This damage is estimated to be Rs.50,000 crores annually. Tomato (Lycopersicon esculentum Mill.) is the second most important vegetable crop next to potato grown in almost all parts of India. It is a rich source of vitamins A and C. The present world production is about 100 million tons of fresh fruit produced on 3.7 million hectare. It is affected by several diseases, wilt of tomato caused by Fusarium oxysporum f. sp. lycopersici (Sacc.) is the
1976; Srinon et al., 2006). It is a devastating disease causing considerable economic losses ranging from 10-80\% yield loss in tomato production (Keshwan and Chaudhary, 1977). Fusarium wilt is soil-borne in nature application of fungicides to control this disease is not very effective. However, the regular use of chemical fungicides is harmful for the environment (Lewis et al., 1996). Hence, there is a need to develop eco-friendly practices for the control of soil borne phytopathogens. Recent trends favor the use of natural plant extracts, biological control agents and several others components. The various types of biological control agents 
such as bacteria and fungi are involved in biocontrol activity. Biological control agents (BCAs) offer an alternative to the chemical based control of fungal phytopathogens as they can kill or limit the growth of pathogens without posing threat to the environment. Various bacteria, fungi and protists are known to have such features. Various mechanisms involved in biocontrol process are: competition for nutrients, secretion of lytic enzymes, secretion of toxic metabolites and direct parasitism on host (Agrios, 2005). Members of the genus Trichoderma are well known BCAs (Elad et al., 1983; Chet, 1987). It has been very effectively used for the control of large number of soil borne plant pathogen like Phytophthora, Rhizoctonia, Sclerotium, Phythium, Fusarium, Sclerotinia, and Galumannomyces. Presently, for commercialization purpose there are mainly three species used viz, Trichoderma harzianum, Trichoderma viride and Trichoderma koningii.

In recent years biological control of soil borne plant pathogens is very popular (Hanafi, 2003; Giotis et al., 2009). Successful reductions of Fusarium wilt in many crops with application of different species of Trichoderma have been found. However, it is also reported that all the isolates of Trichoderma spp. are not equally effective in control of pathogen in vitro and in vivo conditions to control diseases. Therefore, specific isolates are needed for successful control of a particular pathogen.

The main objective of the present investigation is to check the antagonistic potential of Trichoderma isolates against F.o.l under in vitro conditions.

\section{Materials and Methods}

Collection, isolation identification and purification of fungi isolated from rhizospheric and non rhizospheric soil

Soil samples were collected from the rhizosphere soil of different crop niches in Uttar Pradesh (India). Five- fold serial dilutions as described by Singh and Singh, (1970) for each soil sample was prepared in sterilized distilled water and $0.5 \mathrm{ml}$ diluted sample was poured on the surface of Trichoderma Specific Medium (TSM) (Elad et al., 1981). Plates were incubated at $28 \pm$ $2^{\circ} \mathrm{C}$ for $96 \mathrm{~h}$, morphologically different colonies appearing on the plates were purified on Potato Dextrose Agar Medium (PDA) (HiMedia, India)

Test pathogen i. e. Fusarium oxysporum f. sp. lycopersici (ITCC no. 1322) was procured from division of Plant Pathology, IARI, New Delhi.

\section{Morphological identification}

Cultural and morphological observations of colony were based on Trichoderma isolates grown on PDA for 7 days in an incubator at $25 \pm 2{ }^{\circ} \mathrm{C}$ with altering $12 \mathrm{~h} / 12 \mathrm{~h}$ fluorescent light/ darkness. Characters of the conidiumbearing structures and conidia were assessed for each isolate (Table 2).

\section{Antagonistic Activity of Trichoderma Isolates}

The dual culture technique described by Morton and Stroube was used to test the antagonistic ability of 17 isolates of three different Trichoderma spp. viz; T. harzianum, T. viride, T. koningii, against Fusarium oxysporum f.sp.lycopercsi. The pathogen and Trichoderma spp. were grown on PDA for a week at $25 \pm 2{ }^{\circ} \mathrm{C}$. $5 \mathrm{~mm}$ disc of the target fungi cut from the periphery was transferred to the Petri dish previously poured with PDA. Trichoderma spp. was transferred aseptically 
in the same plate of opposite end and were incubated at room temperature with alternate light and darkness for 7 days and observed periodically. Control plates were maintained without Trichoderma. The experiment was replicated thrice and percent growth inhibition was calculated by the formula of $\mathrm{I}=(\mathrm{C}$ 'T $\mathrm{T}) / \mathrm{C}$ $\times 100$, where $\mathrm{C}$ is mycelial growth in control plate, $\mathrm{T}$ is mycelial growth in test organisms inoculated plate and I is inhibition of mycelial growth. Vincent et al., (1999).

\section{Results and Discussion}

A total of 25 soil samples were collected from the different locations of U.P. and M.H. Out the 25 soil samples 17 isolates of Trichoderma were obtained belonging to three different species T.harzianum, T.viride and T.koningii. Microscopic studies were done for the species level identification. Cultural and physiological studies of all the isolates were also done. Antagonistic potential of 17 isolates of three Trichoderma species was determined through dual culture technique (Table 3).

Table.1 Isolation of different Trichoderma isolates

\begin{tabular}{|l|l|c|c|l|}
\hline S.N & Location & District/State & Crop/field & Strain name \\
\hline 1. & Vill-Chirori & Meerut (UP) & Paddy & SVP-Tkoni 1 \\
\hline 2. & P.D.K.V. & Akola (M.H) & Pitunia & SVP-Tkoni 2 \\
\hline 3. & P.D.K.V. & Akola (M.H) & Chrysenthimum & SVP-Tkoni 3 \\
\hline 4. & Anand sagar & Akola(M.H) & Wheat & SVP-Tharz 1 \\
\hline 5. & P.D.K.V. & Akola (M.H) & Cotton & SVP-Tharz 2 \\
\hline 6. & Ag. Collage PDKV & Akola(M.H) & Marigold & SVP-Tharz 3 \\
\hline 7. & P.D.K.V. & Akola (M.H) & Marigold & SVP-Tharz 4 \\
\hline 8. & P.D.K.V. & Akola (M.H) & Marigold & SVP-Tharz 5 \\
\hline 9. & Anandsagar & Akola(M.H) & Bamboo & SVP-Tharz 6 \\
\hline 10 & Ganganagar & Meerut (UP) & Black Gram & SVP-Tharz 7 \\
\hline 11. & Ganganagar & Meerut (U.P) & Garlic & SVP-Tharz 8 \\
\hline 12. & Ganganagar & Meerut (U.P) & Pea & SVP-Tharz 9 \\
\hline 13. & Ganganagar & Meerut (U.P) & Pea & SVP-Tharz 10 \\
\hline 14 & Village Rajpura & Meerut (U.P) & Wheat & SVP-Tharz 11 \\
\hline 15. & Village Rajpura & Meerut (U.P) & Garlic & SVP-Tviri 1 \\
\hline 16. & Village Rajpura & Meerut (U.P) & Sugarcane & SVP-Tviri 2 \\
\hline 17. & Village Rajpura & Meerut (U.P) & Sugarcane & SVP-T \\
\hline
\end{tabular}


Table.2 Morphological descriptors used for characterization of native isolates of Trichoderma spp

\begin{tabular}{|c|c|c|c|c|c|c|c|c|c|c|c|}
\hline \multirow{2}{*}{$\begin{array}{l}\text { Strain } \\
\text { Name }\end{array}$} & \\
\hline & $\begin{array}{l}\text { Colony growth } \\
\text { rate }(\mathrm{cm} / \text { day })\end{array}$ & $\begin{array}{l}\text { Colony } \\
\text { colour }\end{array}$ & $\begin{array}{l}\text { Reverse } \\
\text { Colour } \\
\end{array}$ & $\begin{array}{l}\text { Colony } \\
\text { edge }\end{array}$ & $\begin{array}{l}\text { Mycelial } \\
\text { form }\end{array}$ & $\begin{array}{l}\text { Mycelial } \\
\text { colour }\end{array}$ & Conidiation & $\begin{array}{l}\text { Conidiophore } \\
\text { branching }\end{array}$ & $\begin{array}{l}\text { Conidia } \\
\text { wall }\end{array}$ & \begin{tabular}{|l|}
$\begin{array}{l}\text { Conidial } \\
\text { colour }\end{array}$ \\
\end{tabular} & \begin{tabular}{|l|} 
Chlamydos \\
pores
\end{tabular} \\
\hline $\begin{array}{l}\text { SVP- } \\
\text { Tkoni } 1\end{array}$ & $7-8$ in 3days & $\begin{array}{l}\text { Dirty } \\
\text { green }\end{array}$ & Yellowish & Smooth & $\begin{array}{l}\text { Floccose to } \\
\text { Arachnoid }\end{array}$ & $\begin{array}{l}\text { Watery } \\
\text { white }\end{array}$ & $\begin{array}{l}\text { Ring like } \\
\text { zones }\end{array}$ & $\begin{array}{l}\text { Highly branched, } \\
\text { regular }\end{array}$ & Rough & Green & \begin{tabular}{|l|} 
Not \\
observed
\end{tabular} \\
\hline $\begin{array}{l}\text { SVP- } \\
\text { Tkoni } 2\end{array}$ & 8-9 in 3days & Green & Light yellow & Smooth & $\begin{array}{l}\text { Floccose to } \\
\text { Arachnoid }\end{array}$ & $\begin{array}{l}\text { Watery } \\
\text { white }\end{array}$ & $\begin{array}{l}\text { Ring like } \\
\text { zones }\end{array}$ & $\begin{array}{l}\text { Branched, } \\
\text { regular }\end{array}$ & Rough & Green & \begin{tabular}{|l|} 
Not \\
observed
\end{tabular} \\
\hline $\begin{array}{l}\text { SVP- } \\
\text { Tkoni } 3\end{array}$ & 6-7 in 3days & $\begin{array}{l}\text { Blackish } \\
\text { green }\end{array}$ & $\begin{array}{l}\text { Dark } \\
\text { brownish }\end{array}$ & Smooth & Arachnoid & $\begin{array}{l}\text { Watery } \\
\text { white }\end{array}$ & $\begin{array}{l}\text { Ring like } \\
\text { zones }\end{array}$ & $\begin{array}{l}\text { Branched, } \\
\text { regular }\end{array}$ & Rough & Green & $\begin{array}{l}\text { Not } \\
\text { observed }\end{array}$ \\
\hline $\begin{array}{l}\text { SVP- } \\
\text { Tharz 1 }\end{array}$ & 8-9 in 3days & $\begin{array}{l}\text { Dark } \\
\text { green }\end{array}$ & Colourless & Wavy & $\begin{array}{l}\text { Floccose to } \\
\text { Arachnoid }\end{array}$ & $\begin{array}{l}\text { Watery } \\
\text { white }\end{array}$ & $\begin{array}{l}\text { Ring like } \\
\text { zones }\end{array}$ & $\begin{array}{l}\text { Highly branched, } \\
\text { regular }\end{array}$ & Smooth & Green & \begin{tabular}{|l|} 
Not \\
observed
\end{tabular} \\
\hline $\begin{array}{l}\text { SVP- } \\
\text { Tharz } 2\end{array}$ & 8-9 in 3days & $\begin{array}{l}\text { Green to } \\
\text { dark green }\end{array}$ & Yellowish & Smooth & $\begin{array}{l}\text { Floccose to } \\
\text { Arachnoid }\end{array}$ & $\begin{array}{l}\text { Watery } \\
\text { white }\end{array}$ & $\begin{array}{l}\text { Ring like } \\
\text { zones }\end{array}$ & $\begin{array}{l}\text { Branched, } \\
\text { regular }\end{array}$ & Smooth & Green & $\begin{array}{l}\text { Not } \\
\text { observed }\end{array}$ \\
\hline $\begin{array}{l}\text { SVP- } \\
\text { Tharz } 3\end{array}$ & 8-9 in 3days & $\begin{array}{l}\text { Light } \\
\text { green }\end{array}$ & Colourless & Smooth & $\begin{array}{l}\text { Floccose to } \\
\text { Arachnoid }\end{array}$ & $\begin{array}{l}\text { Watery } \\
\text { white }\end{array}$ & $\begin{array}{l}\text { Ring like } \\
\text { zones }\end{array}$ & $\begin{array}{l}\text { Highly branched, } \\
\text { regular }\end{array}$ & Smooth & Green & \begin{tabular}{|l|} 
Not \\
observed
\end{tabular} \\
\hline $\begin{array}{l}\text { SVP- } \\
\text { Tharz } 4\end{array}$ & 5-6 in 3days & $\begin{array}{l}\text { Snow } \\
\text { white } \\
\text { green }\end{array}$ & Orange & Smooth & Floccose & $\begin{array}{l}\text { Watery } \\
\text { white }\end{array}$ & $\begin{array}{l}\text { Ring like } \\
\text { zones }\end{array}$ & $\begin{array}{l}\text { Branched, } \\
\text { regular }\end{array}$ & Smooth & Green & \begin{tabular}{|l|}
$\begin{array}{l}\text { Not } \\
\text { observed }\end{array}$ \\
\end{tabular} \\
\hline $\begin{array}{l}\text { SVP- } \\
\text { Tharz } 5\end{array}$ & 8-9 in 3days & $\begin{array}{l}\text { Whitish } \\
\text { green }\end{array}$ & Colourless & Smooth & $\begin{array}{l}\text { Floccose to } \\
\text { Arachnoid }\end{array}$ & $\begin{array}{l}\text { Watery } \\
\text { white }\end{array}$ & $\begin{array}{l}\text { Ring like } \\
\text { zones }\end{array}$ & $\begin{array}{l}\text { Branched, } \\
\text { regular }\end{array}$ & Smooth & Green & \begin{tabular}{|l|}
$\begin{array}{l}\text { Not } \\
\text { observed }\end{array}$ \\
\end{tabular} \\
\hline $\begin{array}{l}\text { SVP- } \\
\text { Tharz } 6\end{array}$ & 8-9 in 3days & $\begin{array}{l}\text { Cottony } \\
\text { white } \\
\text { green }\end{array}$ & Yellowish & Smooth & $\begin{array}{l}\text { Floccose to } \\
\text { Arachnoid }\end{array}$ & $\begin{array}{l}\text { Watery } \\
\text { white }\end{array}$ & $\begin{array}{l}\text { Ring like } \\
\text { zones }\end{array}$ & $\begin{array}{l}\text { Branched, } \\
\text { regular }\end{array}$ & Smooth & Green & \begin{tabular}{|l|}
$\begin{array}{l}\text { Not } \\
\text { observed }\end{array}$ \\
\end{tabular} \\
\hline $\begin{array}{l}\text { SVP- } \\
\text { Tharz } 7\end{array}$ & 8-9 in 3days & $\begin{array}{l}\text { Light } \\
\text { green }\end{array}$ & Light yellow & Smooth & $\begin{array}{l}\text { Floccose to } \\
\text { Arachnoid }\end{array}$ & $\begin{array}{l}\text { Watery } \\
\text { white }\end{array}$ & Flat & $\begin{array}{l}\text { Branched, } \\
\text { regular }\end{array}$ & Smooth & Green & \begin{tabular}{|l|} 
Not \\
observed
\end{tabular} \\
\hline $\begin{array}{l}\text { SVP- } \\
\text { Tharz } 8\end{array}$ & $7-8$ in 3days & $\begin{array}{l}\text { Watery } \\
\text { white }\end{array}$ & Colourless & Wavy & Arachnoid & $\begin{array}{l}\text { Watery } \\
\text { white }\end{array}$ & $\begin{array}{l}\text { Ring like } \\
\text { zones }\end{array}$ & $\begin{array}{l}\text { Branched, } \\
\text { regular }\end{array}$ & Smooth & Green & \begin{tabular}{|l|} 
Not \\
observed \\
\end{tabular} \\
\hline $\begin{array}{l}\text { SVP- } \\
\text { Tharz } 9\end{array}$ & 8-9 in 3days & $\begin{array}{l}\text { Snow } \\
\text { white } \\
\text { green }\end{array}$ & Colourless & Smooth & $\begin{array}{l}\text { Floccose to } \\
\text { Arachnoid }\end{array}$ & $\begin{array}{l}\text { Watery } \\
\text { white }\end{array}$ & $\begin{array}{l}\text { Ring like } \\
\text { zones }\end{array}$ & $\begin{array}{l}\text { Branched, } \\
\text { regular }\end{array}$ & Smooth & Green & \begin{tabular}{|l|}
$\begin{array}{l}\text { Not } \\
\text { observed }\end{array}$ \\
\end{tabular} \\
\hline $\begin{array}{l}\text { SVP- } \\
\text { Tharz } 10\end{array}$ & 8-9 in 3days & $\begin{array}{l}\text { Light } \\
\text { green }\end{array}$ & Yellowish & Smooth & $\begin{array}{l}\text { Floccose to } \\
\text { Arachnoid }\end{array}$ & $\begin{array}{l}\text { Watery } \\
\text { white }\end{array}$ & $\begin{array}{l}\text { Ring like } \\
\text { zones }\end{array}$ & $\begin{array}{l}\text { Branched, } \\
\text { regular }\end{array}$ & Rough & Green & \begin{tabular}{|l|} 
Not \\
observed
\end{tabular} \\
\hline $\begin{array}{l}\text { SVP- } \\
\text { Tharz } 11\end{array}$ & 8-9 in 3days & $\begin{array}{l}\text { Snow } \\
\text { white }\end{array}$ & Colourless & Smooth & $\begin{array}{l}\text { Floccose to } \\
\text { Arachnoid }\end{array}$ & $\begin{array}{l}\text { Watery } \\
\text { white }\end{array}$ & Flat & $\begin{array}{l}\text { Highly branched, } \\
\text { regular }\end{array}$ & Smooth & Green & \begin{tabular}{|l|} 
Not \\
observed
\end{tabular} \\
\hline
\end{tabular}




\begin{tabular}{|c|c|c|c|c|c|c|c|c|c|c|c|}
\hline $\begin{array}{l}\text { SVP-Tviri } \\
1\end{array}$ & $8-9$ in 3days & $\begin{array}{l}\text { Dark } \\
\text { green }\end{array}$ & Colourless & Smooth & $\begin{array}{l}\text { Floccose to } \\
\text { Arachnoid }\end{array}$ & $\begin{array}{l}\text { Watery } \\
\text { white }\end{array}$ & $\begin{array}{l}\text { Ring like } \\
\text { zones }\end{array}$ & $\begin{array}{l}\text { Highly branched, } \\
\text { regular }\end{array}$ & Rough & Green & $\begin{array}{l}\text { Not } \\
\text { observed }\end{array}$ \\
\hline $\begin{array}{l}\text { SVP-Tviri } \\
2\end{array}$ & $8-9$ in 3 days & $\begin{array}{l}\text { Greyish } \\
\text { green }\end{array}$ & Colourless & Smooth & $\begin{array}{l}\text { Floccose to } \\
\text { Arachnoid }\end{array}$ & $\begin{array}{l}\text { Watery } \\
\text { white }\end{array}$ & $\begin{array}{l}\text { Ring like } \\
\text { zones }\end{array}$ & $\begin{array}{l}\text { Branched, } \\
\text { regular }\end{array}$ & Rough & Green & $\begin{array}{l}\text { Not } \\
\text { observed }\end{array}$ \\
\hline SVP-T & $8-9$ in 3 days & $\begin{array}{l}\text { Dirty } \\
\text { green }\end{array}$ & $\begin{array}{l}\text { Dark } \\
\text { greenish }\end{array}$ & Smooth & $\begin{array}{l}\text { Floccose to } \\
\text { Arachnoid }\end{array}$ & $\begin{array}{l}\text { Watery } \\
\text { white }\end{array}$ & $\begin{array}{l}\text { Ring like } \\
\text { zones }\end{array}$ & $\begin{array}{l}\text { Ball like } \\
\text { structure }\end{array}$ & Rough & Green & $\begin{array}{l}\text { Not } \\
\text { observed }\end{array}$ \\
\hline
\end{tabular}

Table.3 In vitro antagonistic activity of Trichoderma isolates againsnst Fol

\begin{tabular}{|c|c|c|c|}
\hline \multirow[b]{2}{*}{$\begin{array}{l}\text { Sl } \\
\text { no. }\end{array}$} & \multirow[b]{2}{*}{ Trichoderma sp } & \multicolumn{2}{|l|}{ Growth of $\mathrm{Fol}$ at $72 \mathrm{~h}(\mathrm{~cm})$} \\
\hline & & Mycelial growth & $\%$ inhibition in mycelial growth \\
\hline 1 & SVPT-koni 1 & 1.450 & 72.10 \\
\hline 2 & SVPT-koni 2 & 1.267 & 75.60 \\
\hline 3 & SVPT-koni 3 & 1.467 & 71.70 \\
\hline 4 & SVPT-har 1 & 1.233 & 76.00 \\
\hline 5 & SVPT-har 2 & 1.300 & 75.00 \\
\hline 6 & SVPT-har 3 & 1.300 & 75.00 \\
\hline 7 & SVPT-har 4 & 2.233 & 57.05 \\
\hline 8 & SVPT-har 5 & 1.433 & 72.00 \\
\hline 9 & SVPT-har 6 & 1.433 & 72.4 \\
\hline 10 & SVPT-har 7 & 0.967 & 81.4 \\
\hline 11 & SVPT-har 8 & 1.367 & 73.71 \\
\hline 12 & SVPT-har 9 & 1.367 & 73.71 \\
\hline 13 & SVPT-har 10 & 1.333 & 74.40 \\
\hline 14 & SVPT-har 11 & 1.467 & 71.70 \\
\hline 15 & SVPT-viri 1 & 1.400 & 73.0 \\
\hline 16 & SVPT-viri 2 & 1.167 & 77.5 \\
\hline 17 & SVPT- & 1.733 & 66.60 \\
\hline 18 & Control & 4.20 & \\
\hline & CD@ $9 \%$ & 0.131 & \\
\hline
\end{tabular}


Fig.1 Antagonistic activity of Trichoderma spp. on the Fusarium oxysporum f.sp.lycopersici (cm.).

\begin{tabular}{|l|l|l|l|l|l|}
\hline $\begin{array}{l}\text { Strain } \\
\text { Name }\end{array}$ & SVPT-koni 1 & SVPT-koni 2 & SVPT-koni 3 & SVPT-har 1 & SVPT-har 2 \\
\hline $\begin{array}{l}\text { Mycelium } \\
\text { growth } \\
\text { inhibition } \\
\text { after } 72 \\
\text { hours }\end{array}$ & & & & & \\
\hline $\begin{array}{l}\text { Coiling } \\
\text { observed } \\
\text { under } \\
\text { microscopic }\end{array}$ & & & & & \\
\hline
\end{tabular}

\begin{tabular}{|c|c|c|c|c|c|}
\hline $\begin{array}{l}\text { Strain } \\
\text { Name }\end{array}$ & SVPT-har 3 & SVPT-har 4 & SVPT-har 5 & SVPT-har 6 & SVPT-har 7 \\
\hline $\begin{array}{l}\text { Mycelium } \\
\text { growth } \\
\text { inhibition } \\
\text { after } 72 \\
\text { hours }\end{array}$ & & & & & \\
\hline $\begin{array}{l}\text { Coiling } \\
\text { observed } \\
\text { under } \\
\text { microscopic }\end{array}$ & & & & & \\
\hline
\end{tabular}

\begin{tabular}{|c|c|c|c|c|c|}
\hline $\begin{array}{l}\text { Strain } \\
\text { Name }\end{array}$ & SVPT-har 8 & SVPT-har 9 & SVPT-har 10 & SVPT-har 11 & SVPT-viri 1 \\
\hline $\begin{array}{l}\text { Mycelium } \\
\text { growth } \\
\text { inhibition } \\
\text { after } 72 \\
\text { hours }\end{array}$ & & & & & \\
\hline $\begin{array}{l}\text { Coiling } \\
\text { observed } \\
\text { under } \\
\text { microscopic }\end{array}$ & & & & & \\
\hline
\end{tabular}




\begin{tabular}{|l|c|l|}
\hline $\begin{array}{l}\text { Strain } \\
\text { Name }\end{array}$ & SVPT-viri 2 & SVPT- \\
\hline $\begin{array}{l}\text { Mycelium } \\
\text { growth } \\
\text { inhibition } \\
\text { after } 72 \\
\text { hours }\end{array}$ & & \\
\hline $\begin{array}{l}\text { Coiling } \\
\text { observed } \\
\text { under } \\
\text { microscopic }\end{array}$ & & \\
\hline
\end{tabular}

In the present investigation, screening of the Trichoderma isolates was done against Fusarium oxysporum f. sp. lycopersici, The potential strains were characterized microscopically. Soil borne fungal plant pathogens are causing economically damage whose pathogenic activities are reducing with the use of fungicide application to minimize losses in plant yield, and quality.

So now the scientists exploit the eco-friendly biological methods of disease control through development of non chemical based biocontrol processes. Among fungal antagonists, Trichoderma have been the most commercialized and efficacious inoculants used world over for the control of soil borne fungal plant pathogens (Elad and Kapat, 1999 and Harman, 2010). Biocontrol agents are also known to provide habitat specific suppressive effects.

Several reports indicate that Trichoderma species can effectively suppress Fusarium wilt pathogens (Vipul et al., 2016). Trichoderma species has multiple mechanisms of action, including coparasitism via production of chitinases, 2-1-3 glucanases and 2-1-4 glucanases, antibiotics, competition, solubilisation of inorganic plant nutrients, induced resistance and inactivation of the pathogen's enzymes involved in the infection process (Altomare et al., 1999 and Howel, 2003).

\section{Acknowledgement}

The authors are grateful for the laboratory support provided by Sardar VallabhbhaI Patel University of agriculture and technology modipuram Meerut.

\section{References}

Agrios, G.N. 2000. Plant Pathol., 5th ed. San Diego, CA, USA. Academic Press 2005.

Agrios, G.N., Significance of plant disease, pp. 25-37, In: Agrios, G.N. (Ed.), Plant Pathology. Academic Press, London.

Altomare, C., Norvell, W.A., Bjorkman, T., Harman, G.E. 1999. Solubilization of phosphates and micronutrients by the plant-growth-promoting and biocontrol fungus Trichoderma harzianum Rifai. 1292-22. Appl. Environ. Microbio., 165: 2926-2933

Chet, I. 1987. Trichoderma application, mode of actionand potential as a biocontrol agent of soil borne plantpathogenic fungi. In: (Chet, I. ed.). Innovative approaches to plant diseases control. John Wiley and Sons, New York.

Elad, Y. and Kapat, A. 1999. The role of Trichoderma harzianum protease in the biocontrol of Botrytis cinerea. Europ. J. Plant Pathol., 105: 177- 189.

Elad, Y., I. Chet, Boyle, P., Henis, Y. 1983. Parasitism of Trichoderma spp. on Rhizoctonia solani and Sclerotium rolfsii scanning electron microscopy 
and fluorescence microscopy, Phytopathol., 73: 85-88.

Elad, Y., I. Chet, Henis, Y. 1981. A selective medium for improving quantitative isolation of Trichoderma spp. from soil. Phytoparas., 9: 1 59-67.

Giotis, C., Markelou, E., Theodoropoulou, A., Toufexi, E., Hodson, R., Shotton, P., Shiel, R., Cooper, J., Leifert, C. 2009. Effect of soil amendments and biological control agents (BCAs) on soilborne root disease caused by Pyrenochaetu lycopersici and Verticillium alboatrum in organic greenhouse tomato production systems. Eur. J. Plant Pathol., 123: 387-400.

Hanafi, A. 2003. Integrated Production and Protection in Green-house Tomato in Morocco.p. 192-197. In: "Tomate Sous Abri" Scientific Publication of CTIFL. Editions Centre Technique Interprofessionnel des Fruits et Légumes, $232 \mathrm{pp}$.

Harman. E.G., Herrera-Estrella, H.A., Horwitz, A.B. and Lorito, M. 2010. Trichoderma - from Basic Biology to Biotechnology. Microbiol., 158.

Howell, C.R. 2003. Mechanisms employed by Trichoderma species in the biological control of plant diseases: history and evolution of current concepts. Plant Dis., 89(11): 1195-1200.

Kesavan, V. and B. Chaudhary. Screening for resistance to Fusarium wilt of tomato. SABRO J., 9: 51-65.

Kumar, V., Kumar, A., Srivastava, M., Pandey, S., Shahid, M., Srivastava,
Y.K. and Trivedi, S. 2016. Trichoderma harzianum (Th. azad) as a Mycoparasite of Fusarium and growth enhancer of Tomato in Glasshouse Conditions. $J$. Pure and Appl. Microbiol., 10: 14631468.

Lewis, J.A., Lumsden, R.D., Locke, J.C. 1996. Biocontrol of dampingoffdiseases caused by Rhizoctonia solani and Pythium ultimum withalginate prills of Gliocladium virens, Trichoderma hamatum andvarious food bases. Biocontrol Sci. Technol., 6: 163-173.

Rick, C.M., Simmonds, N.W. Evaluation of Tomato Crop Plant,.(Ed.), Longman. In: New York, Pp. 263-273,.

Singh, R.S., Singh, N. 1970. Effect of oil cake amendment of soil on population of some wilt causing species of Fusarium. Phytopath. Zei. Schrift.,, 26: 160-167.

Srinon, W., Chuncheen, K., Jirattiwarutkul, K., Soytong, K. \& Kanokmedhakul, S. 2006. Efficacies of antagonistic fungi against Fusarium wilt disease of cucumber and tomatoand the assay of its enzyme activity. J. Agri. Technol., 2(2): 191-201.

Vincent, I., Benhamon, N. and Chet, I. 1999. Induction of defence response in cucumber plants (cucumis sativas L.) by the biocontrol agent Trichoderma harzianum. App. Env. Microbiol., 55: 1061- 1070.

\section{How to cite this article:}

Akash Tomar, Lakshman Prasad, Abhishek Mishra and Sushma Sagar. 2017. Antagonistic Action of Trichoderma Isolates against Fusarium oxysporum lycopersci. Int.J.Curr.Microbiol.App.Sci. 6(2): 258-265. doi: http://dx.doi.org/10.20546/ijcmas.2017.602.031 